\title{
BMJ Open Effect of exercise on symptoms of premenstrual syndrome in low and middle-income countries: a protocol for systematic review and meta-analysis
}

\author{
Pratik Pokharel (D) , 1,2,3 Juwel Rana, ${ }^{4,5}$ Jude Moutchia, ${ }^{2,3}$ Shreeshti Uchai, ${ }^{2,3}$ \\ Aldiona Kerri, ${ }^{2,3}$ Patricia Lorena Luna Gutiérrez, ${ }^{6}$ Rakibul M Islam ${ }^{7}$
}

To cite: Pokharel P, Rana J, Moutchia J, et al. Effect of exercise on symptoms of premenstrual syndrome in low and middle-income countries: a protocol for systematic review and meta-analysis. BMJ Open 2020;10:e039274. doi:10.1136/ bmjopen-2020-039274

- Prepublication history and additional material for this paper are available online. To view these files, please visit the journal online (http://dx.doi. org/10.1136/bmjopen-2020039274).

Received 13 April 2020 Revised 22 July 2020 Accepted 17 August 2020

Check for updates

(C) Author(s) (or their employer(s)) 2020. Re-use permitted under CC BY-NC. No commercial re-use. See rights and permissions. Published by BMJ.

For numbered affiliations see end of article.

Correspondence to

Pratik Pokharel;

pokharelpratik1921@gmail.com

\section{ABSTRACT}

Introduction Premenstrual syndrome (PMS) has the potential to affect the quality of life adversely. Published guidelines recommend the use of exercise as part of the first-line management interventions for PMS. However, the published evidence related to the effectiveness of physical activity and PMS is inconclusive. This review will assess the effectiveness of exercise-based interventions in reducing PMS in women screened or diagnosed with PMS in low and middle-income countries, where the prevalence of PMS is high.

Methods and analysis Electronic databases will be researched, including Embase, Cochrane Central Register of Controlled Trials, MEDLINE, PsycINF0, Web of Science, ClinicalTrials.gov and Google Scholar. All the studies published until March 2020 will be included. A standardised data extraction form will be used adapted from the Cochrane Handbook of Systematic Reviews of Interventions. Included articles will be assessed using the risk of bias tools based on study design. Data will be analysed using Review Manager V.5.3. The inversevariance random-effects method will be used to report the standardised mean difference. A meta-analysis will be used only if studies are sufficiently homogenous. A narrative synthesis will be undertaken when studies are heterogeneous. Methodological heterogeneity between studies will be evaluated by considering the study types. Statistical heterogeneity will be tested using the $I^{2}$ test. Subgroup analyses may be performed only for the primary outcome in case of sufficient studies. Sensitivity analysis will be conducted to assess the impact of intervention excluding studies without randomisation and studies with a high risk of bias. Funnel plots will be used to assess the potential reporting bias and small-study effects only when there are more than 10 studies included in the metaanalysis.

Ethics and dissemination This study does not require ethical approval, as the review is entirely based on published studies. The results will be published and/or will be presented at a pertinent conference.

PROSPERO registration number CRD42020163377.

\section{INTRODUCTION}

Premenstrual syndrome (PMS) is a menstrual disorder characterised by repetitive physical,
Strengths and limitations of this study

$\Rightarrow$ This systematic review will provide robust evidence related to the effectiveness of exercise-based interventions in reducing premenstrual syndrome (PMS) symptoms in women screened or diagnosed with PMS in low and middle-income countries (LMICs).

$\Rightarrow$ The findings of this systematic review will provide an important reference for stakeholders and clinicians from LMICs when treating patients with PMS.

$\Rightarrow$ Since articles with relevant information published in languages other than English will be excluded, it may lead to publication bias.

$\Rightarrow$ We may not be able to undertake anticipated subgroup analyses due to the range of different types of exercise, duration, intensity, and so on.

behavioural and psychological symptoms. It usually starts 5-7 days before menstruation (at the end of the secretory phase of the menstrual cycle) and rapidly resolves $2-4$ days after menstruation (in the follicular phase). ${ }^{1}$ Globally, $90 \%$ women of reproductive age experience several premenstrual symptoms while 20\%-40\% experience PMS, ${ }^{2}$ with the highest prevalence reported in low and middle-income countries (LMICs). A systematic review reported $30 \%-99.5 \%$ prevalence of PMS in Iran, ${ }^{3}$ while a recent study in Nepal reported that $72 \%$ of university students had at least one symptom of PMS. ${ }^{4}$

The common clinical and psychological symptoms of PMS include depression, mood fluctuations, nervousness, irritability, fatigue, overeating, weight gain, breast tenderness, muscle and joint pain, abdominal bloating, diarrhoea and performance reduction. ${ }^{5-7}$ Most study findings show that $5 \%-8 \%$ of women have mild to severe symptoms while some studies estimate $20 \%$ of all fertile women experience clinically significant premenstrual complaints. ${ }^{8}$ Although the 
aetiology of PMS remains unclear, ${ }^{9-12}$ the prevalence of PMS is higher in women who do not exercise, are obese and perform poorly in academics while the prevalence is lower among hormonal contraception users. ${ }^{11}$ Therefore, PMS as a distressing experience of women needs attention when trying to achieve a healthy life and gender equality in resource-poor settings.

The current management of PMS mostly targets symptom relief that includes non-pharmacological treatment, surgery and medication. ${ }^{13}$ The combination of oral contraceptives, use of selective serotonin reuptake inhibitors (SSRIs) and gonadotropin-releasing hormone analogues are major pharmacological methods. Nonpharmacological methods include lifestyle modification, cognitive-behavioural therapy (CBT) and dietary supplementation. ${ }^{8121415}$ Although SSRIs are the first-line management option for PMS, side effects are evident. ${ }^{16}$ A study found that aerobic exercise improves PMS symptoms in women by reducing the serum aldosterone and increasing oestrogen and progesterone. ${ }^{17}$ Physical exercise has minimal risks or side effects and is a suitable management tool in the context of LMICs. Therefore, attention has been paid to non-drug treatments, particularly physical activity. ${ }^{18}$ The National Institute for Health and Care Excellence guideline in the UK suggests lifestyle modifications such as exercise and balanced meals for management of mild to severe PMS along with other medications. ${ }^{19}$ Likewise, the Royal College of Obstetricians and Gynaecologists recommends exercise and CBT as first-line management for PMS. ${ }^{20}$

Several systematic reviews on PMS have been published but their main focus was limited to CBT, acupuncture, dietary supplementation and herbal remedies. ${ }^{9}$ 21-23 Some physicians recommend exercise as an intervention but emphasise it as non-evidence-based therapy. ${ }^{12} 14$ Lack of a well-organised review for healthcare providers increases the burden of proposing scientifically verified management for PMS. Furthermore, there is no systematic review on the effectiveness of physical exercise on PMS in the context of LMICs where PMS poses a great burden. This systematic review and meta-analysis, therefore, will assess the effectiveness of exercise-based interventions in reducing PMS symptoms in women screened or diagnosed with PMS in LMICs.

\section{METHODS AND ANALYSIS}

The planned study was conceptualised in December 2019 and anticipated to end in November 2020.

\section{Protocol registration}

This systematic review protocol was registered with the International Prospective Register of Systematic Reviews by following the guidelines. The protocol of this systematic review aligns with the Preferred Reporting Items for Systematic Review and Meta-Analysis Protocols, ${ }^{24}$ along with the recommendations adopted from the metaanalysis of observational studies in epidemiology group. ${ }^{25}$

\section{Inclusion and exclusion criteria}

We will include studies on women of reproductive age (15-49 years) who meet either the screening or diagnostic criteria for PMS. Validated symptom screening tool such as premenstrual symptoms screening tool (PSST) or established diagnostic tool such as Diagnostic and Statistical Manual of Mental Disorders, Fourth Edition should have been used for inclusion.

We will exclude studies that included women who are either pregnant or of non-reproductive age. We will also exclude studies on reproductive-aged women with psychiatric disorders and chronic diseases like breast cancer and diabetes to reduce misclassification of symptoms due to other causes except PMS. Also, we will exclude studies on women who are on any medication or mineral supplements for PMS and non-English studies. Studies carried out in any high-income country setting and studies with interventions comparing two different exercises without a control arm will also be excluded. Countries will be classified as low, middle or high income according to World Bank's classification criteria. ${ }^{26}$

\section{Intervention}

A predefined intervention for this review is exercise, which was defined for this review as "planned, structured and repetitive bodily movement done to improve or maintain one or more components of physical fitness'. ${ }^{27}$ The intervention can include any form of cardio or resistance exercise or a combination of both for at least 6 weeks. Interventions like yoga will also be included as it includes physical exercise (asanas) primarily and has been shown to reduce symptoms of menstrual distress. ${ }^{28}$ Hence, no limitation will be applied for exercise type. Studies with a combination of interventions like factorial trials or non-inferiority trials with multiple parallel arms where exercise intervention can be considered as the experimental variable will be included for this review.

\section{Comparator}

The studies using no intervention or usual care, or no treatment or placebo will be considered as comparators in this review.

\section{Outcome}

Primary outcome

The studies with the outcome reported as total or overall PMS symptom score measured by any validated screening tool or questionnaire (eg, PSST questionnaire) will be the primary outcome in this review.

\section{Secondary outcome}

The secondary outcome will be the quality of life score and accumulated score for physical, behavioural, emotional or psychological symptoms, as measured by validated tools. Studies reporting only secondary outcomes will also be considered for inclusion. 


\section{Study design}

We will include randomised controlled trials (RCT; parallel, cluster or individual), quasiexperimental studies and cohort studies.

\section{Setting}

The studies conducted in the community, clinical or hospital settings in low-income, lower middle-income and upper middle-income countries will be included.

\section{Patient and public involvement}

No patient is involved in either the design or planning phase of this study.

\section{Data sources and search strategy}

Multiple databases will be searched for the relevant research articles which include Embase, Cochrane Central Register of Controlled Trials, MEDLINE, PsycINFO, Web of Science, ClinicalTrials.gov and Google Scholar. The search strategy for this review will be developed by adapting, expanding, blending and updating search strategies from Cochrane Gynaecology and Fertility search strategies. ${ }^{29}$ The search strategy will use the search terms presented in the online supplemental file 1 . The researched articles will then be limited using the filters for LMICs and the English language. All the eligible studies published until March 2020 will be included. A search strategy developed for MEDLINE is shown in the online supplemental file 1 . This search will be updated until the above-mentioned timeline.

\section{Selection of studies}

All the retrieved search results will be stored in an EndNote library. The duplicates will be removed and stored in a separate library. Titles followed by the abstracts of the studies retrieved from the search and other sources will be screened independently by two review authors (SU and PLLG) to identify relevant studies meeting the inclusion criteria. The full text of the selected studies will then be subsequently reviewed by two independent reviewers (AK and JM) for eligibility. Discrepancies, if any, will be resolved through discussion with the other two reviewers (PP and JR) and the explanations for the decisions taken with regard to inclusion or exclusion of studies will be well documented as per Preferred Reporting Items for Systematic Reviews and Meta-Analyses reporting guidelines for systematic reviews. ${ }^{30}$

\section{Data extraction}

A standard data extraction form will be adapted from the Cochrane Handbook of Systematic Reviews of Interventions. ${ }^{31}$ The data extraction will be carried out independently by three reviewers (SU, AK and PLLG) after piloting. The following data will be extracted from each selected study: author and year; the economic level of the country; study design; start date and end date; study duration; ethical approval obtained; population description; setting; sample size; lost to follow-up; PMS diagnosis method, time postdiagnosis of PMS; comorbidities; intervention type; timing or frequency of intervention; intervention provider and provider settings; compliance; time points measured and reported; questionnaire used; primary and secondary outcomes reported.

The authors will be contacted in case of missing data. In studies where SDs are lacking, imputation may be done considering the similarity of the scale used, intervention and sample size and effect size. This will be done in agreement among the authors of the review.

\section{Risk of bias and quality assessment}

The Cochrane risk of bias tool ${ }^{32}$ will be used for quality assessment in RCTs while the Risk of Bias in Nonrandomized Studies of Interventions ${ }^{33}$ will be used to assess the risk of bias in quasiexperimental studies and cohort studies. The assessment will be performed independently by three reviewers (JM, SU and PP) and discrepancies resolved by consulting other reviewers (JR and RMI) when necessary. Risk of bias graph and summary will be generated.

\section{Statistical analysis}

\section{Data synthesis}

Data will be analysed using Review Manager V.5.3. As the primary outcome of this intervention is usually measured on a continuous scale, we will synthesise data retained from primary studies by meta-analysis using an inverse-variance random-effects method to report the standardised mean difference (SMD). The SMD will be used because we anticipate the PMS symptom scores to be reported using different tools such as the PSST or Penn Daily Symptom Report. The random-effects method will be used for this analysis to allow for between-study differences in intervention effects.

A meta-analysis will be used only if the studies are sufficiently homogenous. If studies are not sufficiently homogenous $\left(\mathrm{I}^{2}\right.$ statistic $>75.0 \%$ ), we will adopt a narrative synthesis. After discussing the baseline characteristics, types of intervention and outcomes considered in the studies, we will describe patterns across studies in terms of the magnitude and direction of any observed effects. We will consider factors that may account for conflicting results; considering the complexity and heterogeneity of assessing psychological and behavioural outcomes. We will present the data on tables; including information on participants, intervention, outcome measures, and magnitude and direction of effects. We will use vote counting cautiously to conclude, taking into account the sample size of the studies. Three authors forming two groups (JM, SU and AK) and (PP, JR and PLLG) will synthesise data separately and discrepancies will be resolved by discussion with RMI.

\section{Assessment of heterogeneity}

Clinical heterogeneity will be evaluated by considering differences in participants (such as age, parity and occupation), type of intervention (such as jogging, swimming and yoga) and outcomes considered (such as physical, 
psychological and behavioural symptoms) across studies. Study methodological heterogeneity between studies will be evaluated by considering the study types (RCTs vs nonRCTs). Statistical heterogeneity will be tested using the $\mathrm{I}^{2}$ statistic to quantify discrepancy across studies. $\mathrm{I}^{2}$ statistic of $75 \%$ and more indicates significant heterogeneity between studies. ${ }^{34}$ We will explain any observed heterogeneity using subgroup and/or sensitivity analysis.

\section{Subgroup analysis}

Subgroup analyses may be performed only for the primary outcome in case of sufficient studies. Subgroup analyses may be conducted based on the economic level of the country, for example, low-income countries versus lower middle-income and upper middle-income countries. Other subgroup analyses may be carried out by categorising exercise intervention into cardio and strength training or mixed depending on sufficient study types.

\section{Sensitivity analysis}

We will conduct a sensitivity analysis to assess the impact of excluding studies without randomisation (non-RCTs). We will also conduct sensitivity analyses to assess the impact of exercise by excluding studies with a high risk of bias.

\section{Assessment of reporting biases}

Funnel plots will be used to assess the potential reporting bias and small-study effects only when there are more than 10 studies in the meta-analysis. The quantification of publication bias will be done using Egger's regression test method. ${ }^{36}$

\section{Confidence in cumulative evidence}

The studies included in the review will be critically appraised and synthesised in terms of methodological quality along with the relevance, strength and limitations of the evidence presented. The similarities and differences between the studies in terms of their characteristics, design and execution will be identified and their potential impact on study outcome will be explored. The results of the review will be interpreted only after evaluating the quality of the evidence (Grading of Recommendations Assessment, Development and Evaluation) and its pertinence.

\section{DISCUSSION}

We have proposed a systematic review with a transparent and reproducible methodology to assess the effectiveness of exercise-based interventions in the management of PMS in LMICs. The strengths of this review include well-defined study and report characteristics of studies to be included, well-defined data sources, search strategy, data extraction and management and quality assessment procedures, and predefined data synthesis strategies. Potential limitations of this review include the scarcity of RCTs involving exercise-based interventions for PMS in LMICs, the methodological quality of available studies and the possibility of publication bias. Also, potential heterogeneity of exercise-based interventions, measurement tools and types of outcomes evaluated may hinder ease of data synthesis.

As clinicians strive to mitigate the physical and psychological burden of PMS in LMICs, our findings will provide a basis for practice guidelines on the use of low-cost exercise-based interventions in relieving PMS symptoms.

\section{Ethics and dissemination}

This study does not require ethical approval, as the review is entirely based on published studies and there is no human or animal interaction involved.

The findings of the review will be published in a suitable peer-review journal and will also be disseminated in relevant gynaecology and obstetrics conferences drawing stakeholders and clinicians from LMICs.

\section{Author affiliations}

${ }^{1}$ Forum for Health Research and Development, Dharan, Nepal

${ }^{2}$ School of Health and Related Research, The University of Sheffield, Sheffield, UK

${ }^{3}$ École des hautes études en santé publique (EHESP), Paris, France

${ }^{4}$ Department of Public Health, North South University, Dhaka, Bangladesh

${ }^{5}$ Department of Biostatistics and Epidemiology, University of Massachusetts

Amherst, Amherst, Massachusetts, USA

${ }^{6}$ Escuela Andaluza de Salud Pública, Granada, Spain

${ }^{7}$ Women's Health Research Program, Monash University, Melbourne, Victoria, Australia

Contributors PP is the guarantor of this review and conceived the review. PP, $\mathrm{JR}, \mathrm{RMI}$ and $\mathrm{JM}$ designed the review further while PP and RMI contributed to the development of the search strategy. PP, JM, SU, AK and PLLG were involved in the initial draft of the manuscript. JR and RMI were involved in subsequent draft manuscript reviews. All authors edited, modified and approved the final version of this protocol. All authors will contribute equally to data extraction, synthesis and drafting of the final review.

Funding The authors have not declared a specific grant for this research from any funding agency in the public, commercial or not-for-profit sectors.

Competing interests None declared.

Patient consent for publication Not required.

Provenance and peer review Not commissioned; externally peer reviewed.

Open access This is an open access article distributed in accordance with the Creative Commons Attribution Non Commercial (CC BY-NC 4.0) license, which permits others to distribute, remix, adapt, build upon this work non-commercially, and license their derivative works on different terms, provided the original work is properly cited, appropriate credit is given, any changes made indicated, and the use is non-commercial. See: http://creativecommons.org/licenses/by-nc/4.0/.

ORCID iD

Pratik Pokharel http://orcid.org/0000-0001-5815-5927

\section{REFERENCES}

1 Hasani N, Kazemi M, Karimi Afshar $\mathrm{H}$, et al. Comparison of the effects of relaxation and vitamin B6 on emotional and physical symptoms in premenstrual syndrome. Evidence Based Care 2015;5:75-83.

2 Chumpalova P, lakimova R, Stoimenova-Popova M, et al. Prevalence and clinical picture of premenstrual syndrome in females from Bulgaria. Ann Gen Psychiatry 2020;19:3.

3 Ranjbaran M, Omani Samani R, Almasi-Hashiani A, et al. Prevalence of premenstrual syndrome in Iran: a systematic review and metaanalysis. Int $J$ Reprod Biomed 2017;15:679-86.

4 Shrestha DB, Shrestha S, Dangol D, et al. Premenstrual syndrome in students of a teaching hospital. J Nepal Health Res Counc 2019;17:253-7. 
5 Seedhom AE, Mohammed ES, Mahfouz EM. Life style factors associated with premenstrual syndrome among El-Minia university students, Egypt. ISRN Public Health 2013;2013:1-6.

6 Choi D, Lee D-Y, Lehert P, et al. The impact of premenstrual symptoms on activities of daily life in Korean women. J Psychosom Obstet Gynaecol 2010;31:10-15.

7 Silva CM, Gigante DP, Carret ML, et al. Population study of premenstrual syndrome. Public Health J 2006;40:47-56.

8 Yonkers KA, O'Brien PMS, Eriksson E. Premenstrual syndrome. Lancet 2008;371:1200-10.

9 Armour M, Ee CC, Hao J, et al. Acupuncture and acupressure for premenstrual syndrome. Cochrane Database Syst Rev 2018;8:CD005290.

10 Ryu A, Kim T-H. Premenstrual syndrome: a mini review. Maturitas 2015;82:436-40.

11 Panay N. Management of premenstrual syndrome. J Fam Plann Reprod Health Care 2009;35:187-94.

12 Biggs WS, Demuth RH. Premenstrual syndrome and premenstrual dysphoric disorder. Am Fam Physician 2011;84:918-24.

13 Samadi Z, Taghian F, Valiani M. The effects of 8 weeks of regular aerobic exercise on the symptoms of premenstrual syndrome in nonathlete girls. Iran J Nurs Midwifery Res 2013;18:14.

14 Walsh S, Ismaili E, Naheed B, et al. Diagnosis, pathophysiology and management of premenstrual syndrome. Obstet Gynecol 2015;17:99-104.

15 Jarvis $\mathrm{Cl}$, Lynch AM, Morin AK. Management strategies for premenstrual syndrome/premenstrual dysphoric disorder. Ann Pharmacother 2008;42:967-78.

16 Ismaili E, Walsh S, O'Brien PMS, et al. Fourth consensus of the International Society for premenstrual disorders (ISPMD): auditable standards for diagnosis and management of premenstrual disorder. Arch Womens Ment Health 2016;19:953-8.

17 Dehnavi ZM, Jafarnejad F, Kamali Z. The effect of aerobic exercise on primary dysmenorrhea: a clinical trial study. J Educ Health Promot 2018;7:3.

18 Taylor D. From "It's All in Your Head" to "Taking Back the Month": premenstrual syndrome (PMS) research and the contributions of the society for menstrual cycle research. Sex Roles 2006;54:377-91.

19 National Institute for Health and Care Excellence (NICE). Premenstrual syndrome. NICE, 2014. https://cks.nice.org.uk

20 Royal College of Obstetricians and Gynaecologists (RCOG). Management of premenstrual syndrome. BJOG 2016:124:e73-105.

21 Stevinson C, Ernst E. Complementary/alternative therapies for premenstrual syndrome: a systematic review of randomized controlled trials. Am J Obstet Gynecol 2001;185:227-35.

22 Lustyk MKB, Gerrish WG, Shaver S, et al. Cognitive-behavioral therapy for premenstrual syndrome and premenstrual dysphoric disorder: a systematic review. Arch Womens Ment Health 2009;12:85-96.

23 Busse JW, Montori VM, Krasnik C, et al. Psychological intervention for premenstrual syndrome: a meta-analysis of randomized controlled trials. Psychother Psychosom 2009;78:6-15.

24 Shamseer L, Moher D, Clarke M, et al. Preferred reporting items for systematic review and meta-analysis protocols (PRISMA-P) 2015 . elaboration and explanation. BMJ 2015;349:g7647.

25 Stroup DF, Berlin JA, Morton SC, et al. Meta-analysis of observational studies in epidemiology: a proposal for reporting JAMA 2000;283:2008-12.

26 The World Bank Group. World bank country and lending groups World bank data help desk [online]. Available: https://datahelpdesk. worldbank.org/knowledgebase/articles/906519-world-bank-countryand-lending-groups [Accessed $18 \mathrm{Jul} 2020$ ].

27 Caspersen CJ, Powell KE, Christenson GM. Physical activity, exercise, and physical fitness: definitions and distinctions for healthrelated research. Public Health Rep 1985;100:126-31.

28 Oates J. The effect of yoga on menstrual disorders: a systematic review. J Altern Complement Med 2017;23:407-17.

29 Cochrane Gynaecology and Fertility. Resources search strategies CGF. Available: https://cgf.cochrane.org/resources [Accessed Dec 2019].

30 Moher D, Liberati A, Tetzlaff J, et al. PRISMA group: methods of systematic reviews and meta-analysis: preferred reporting items for systematic reviews and meta-analyses: the PRISMA statement. $J$ Clin Epidemiol 2009;62:1006-12.

31 Li T, Higgins JP, Deeks JJ. Chapter 5: Collecting data. In: Cochrane handbook for systematic reviews of interventions. London: Cochrane, 2019.

32 Higgins JPT, Altman DG, Gøtzsche PC, et al. The Cochrane collaboration's tool for assessing risk of bias in randomised trials. BMJ 2011;343:d5928-9.

33 Sterne JA, Hernán MA, McAleenan A, et al. Assessing risk of bias in a non-randomized study. In: Higgins JPT, Thomas J, Chandler $\mathrm{J}$, et al, eds. Cochrane handbook for systematic reviews of interventions, 2019: 621-41. https://training.cochrane.org/handbook/ current/chapter-25\#section-25-3

34 Deeks JJ, Higgins JP, Altman DG, Cochrane Statistical Methods Group. Analysing data and undertaking meta-analyses. In: Higgins JPT, Thomas J, Chandler J, et al, eds. Cochrane handbook for systematic reviews of interventions, 2019: 241-84. https://training. cochrane.org/handbook/current/chapter-10\#section-10-10

35 Higgins JPT, Thompson SG, Deeks JJ, et al. Measuring inconsistency in meta-analyses. BMJ 2003;327:557-60.

36 Lin L, Chu H. Quantifying publication bias in meta-analysis. Biometrics 2018;74:785-94. 